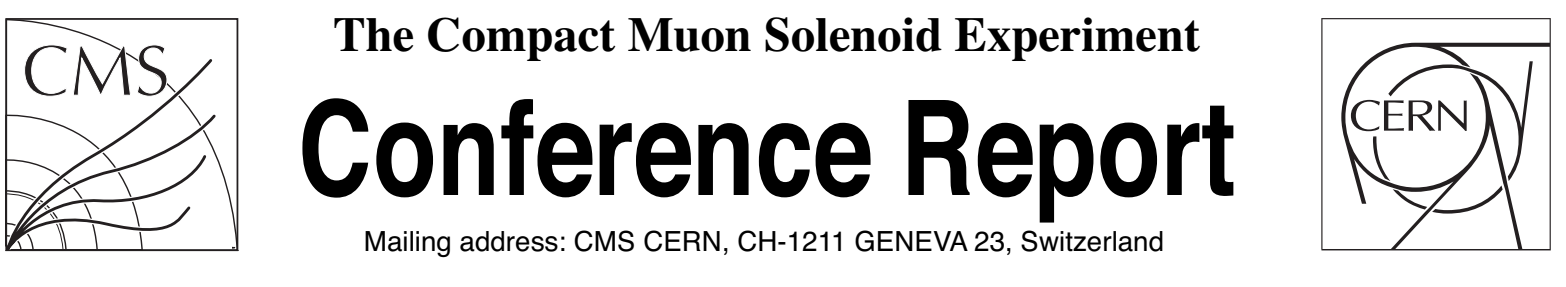

11 September 2019 (v4, 08 November 2019)

\title{
The CMS Muon System performance during the LHC Run-2
}

Nicola Pozzobon for the CMS Collaboration

\begin{abstract}
A highly performing muon system has been fundamental to achieve many of the physics results obtained by CMS during the LHC Run-2. The CMS muon spectrometer presently consists of three detector technologies covering different regions of pseudorapidity. Drift Tube (DT) chambers equip the CMS muon system barrel, whereas Cathode Strip Chambers (CSC) are installed at the CMS endcaps; both are used for offline tracking and provide trigger capabilities. In addition, Resistive Plate Chambers (RPC) complement DT and CSC in both barrel and end-caps, and are mostly used in the trigger. Finally, at different stages of the CMS upgrade programme, the end-caps of the muon spectrometer will be equipped with multiple layers Gas Electron Multiplier (GEM) chambers. A slice test consisting of 10 GEM chambers was successfully operated in 2018, in parallel to the rest of the muon system, to gain experience in view of the installation of the first complete layer of GEM, planned to happen during the second LHC long shutdown (LS2). In this report, the performance of the different detectors comprising the CMS muon system, evaluated using data collected at a centre-of-mass energy of $13 \mathrm{TeV}$ during the LHC Run-2, will be presented, together with the one of the muon trigger. The experience from the integration and commissioning of the GEM slice tests will also be discussed, and the status and plans toward the installation of the first complete layer of GEM detector, happening over LS2, will be highlighted.
\end{abstract}




\title{
The CMS Muon System: performance during the LHC Run-2
}

\author{
N. Pozzobon ${ }^{a, b}$ on behalf of the CMS Collaboration \\ ${ }^{a}$ Università degli Studi di Padova, \\ Dipartimento di Fisica e Astronomia "G. Galilei”, \\ via F. Marzolo, 8, Italy 35131 \\ ${ }^{b}$ INFN, Sezione di Padova, \\ via F. Marzolo, 8, Italy 35131 \\ E-mail: nicola.pozzobon@pd.infn.it
}

\begin{abstract}
A highly performing muon system has been fundamental to achieve many of the physics results obtained by CMS during the LHC Run-2. The CMS muon spectrometer presently consists of three detector technologies covering different regions of pseudorapidity. Drift Tube (DT) chambers equip the CMS muon system barrel, whereas Cathode Strip Chambers (CSC) are installed at the CMS endcaps; both are used for offline tracking and provide trigger capabilities. In addition, Resistive Plate Chambers (RPC) complement DT and CSC in both barrel and endcaps, and are mostly used in the trigger. Finally, at different stages of the CMS upgrade programme, the endcaps of the muon spectrometer will be equipped with multiple layers Gas Electron Multiplier (GEM) chambers. A slice test consisting of 10 GEM chambers was successfully operated in 2018, in parallel to the rest of the muon system, to gain experience in view of the installation of the first complete GEM layer, planned to happen during the second LHC long shutdown (LS2). In this report, the performance of the different detectors comprising the CMS muon system, together with the muon trigger performance, evaluated using data collected at a centre-of-mass energy of $13 \mathrm{TeV}$ during the LHC Run-2, will be presented. The experience from the integration and commissioning of the GEM slice tests will also be discussed, and the status and plans towards the installation of the first complete layer of GEM detector, happening over LS2, will be highlighted.
\end{abstract}

KeYwords: Muon spectrometers, Particle tracking detectors (Gaseous detectors), Trigger detectors 


\section{Contents}

1 Overview 1

2 Muon detectors efficiency and resolution 2

3 Performance of muon triggers during Run-2 5

4 GEM slice test during Run-2 5

5 Conclusions 6

\section{Overview}

The Compact Muon Solenoid (CMS) experiment at the CERN Large Hadron Collider (LHC) is a multi-purpose detector designed for efficient identification and precise measurement of muons [1]. In fact, muons provide a clear signature for final states over a wide momentum range, from a few $\mathrm{GeV}$ to the $\mathrm{TeV}$ scale. Muon candidates from the different CMS sub-detectors also provide fast and efficient triggers exploited for the whole physics program of the experiment, from the observation and measurement of the Higgs boson [2], to the search for supersymmetric particles, from precision measurements in the electroweak sector, to the observation of rare decays in the heavy-flavour sector [3].

The CMS muon system is composed of four different subsystems, as shown in Fig. 1: 250 Drift Tubes (DT) chambers in the central region up to pseudorapidity $|\eta|<1.2$, used for both tracking and trigger, each composed of $8 r \phi$-layers, where $\phi$ is the azimuthal angle, and $4 r z$-layers of drift tubes, 540 Cathode Strip Chambers (CSC) in the forward region, $0.9<|\eta|<2.4$, used for both tracking and trigger, each composed of 6 layers of multi-wire proportional chambers with cathode strip readout, 480+576 trigger-oriented Resistive Plate Chambers (RPC), up to $|\eta|<1.9$, being double-gap RPCs in avalanche mode. The fourth sub-system was introduced in 2017 as the very first Phase-II Upgrade being commissioned with a slice test: Gas Electron Multipliers (GEMs) extending tracking and trigger capabilities in the forward region, $1.6<|\eta|<2.4$ [4].

The performance of the muon system is evaluated in terms of efficiency and resolution of muon hits and muon track segments, i.e. at the level of single channel or single chamber [5]. Muon hits are defined by the reconstructed crossing position of the muon track in the reference frame of a single detector layer, starting from the primary signal ionisation charge collected by wires and strips. Muon hits are one-dimensional in DT chambers, where they are reconstructed after a conversion of the calibrated charge collection time, as well as in RPC, where reconstruction requires strip clustering because of induced charge sharing. On the other hand, muon hits are two-dimensional in CSC, where the two coordinates are read from wires and strips. A muon track segment is reconstructed combining hits in neighboring layers using an assumed straight line fit. Since multiple layers are required to build a track segment, this operation is not available for RPC detectors. 


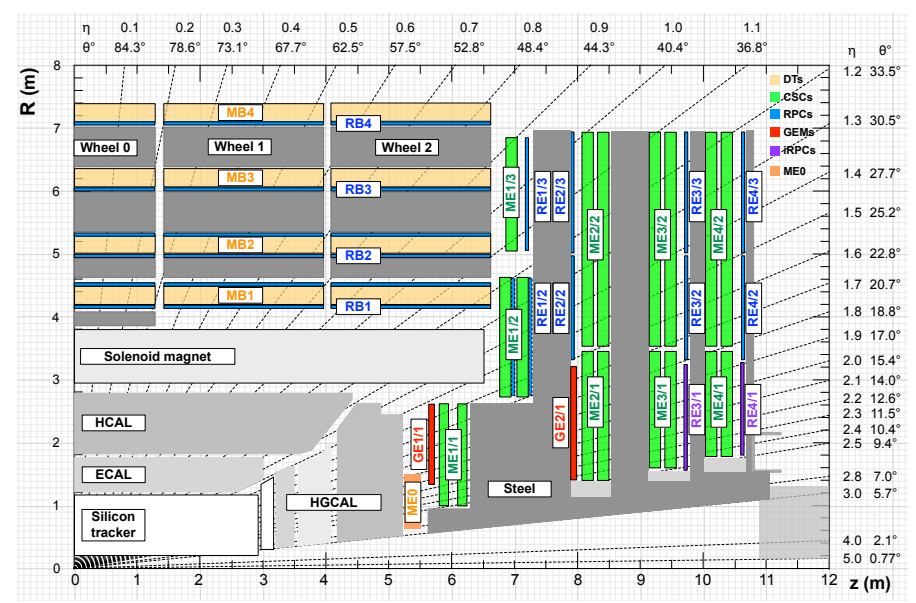

Figure 1. Muon detectors in the CMS experiment [6]: DT chambers are shown in yellow, CSC in green, RPC in blue. New detectors being installed in view of Phase-II upgrades are shown as well: GEM in red and orange, and iRPC in purple. A small number of GEM detectors in GE1/1 were installed in 2017 and have been operational since then.

The efficiency is measured with a data-driven technique called "tag-and-probe", exploiting opposite-sign muon pairs from Drell-Yan or $\mathrm{Z}$ events. One well-reconstructed triggering muon, called the "tag", meeting tight selection criteria, is used to select the event, while another muon, either a tracker muon or a muon track, called the "probe", passing a looser identification, is propagated to fiducial regions of all compatible chambers, searching for close-by segments or hits. The hit efficiency is then measured as the fraction of recorded hits out of the total number of expected hits from the propagated probe, while the segment efficiency is measured as the fraction of segments found in the search window out of the total number of segment projections within the fiducial region. The resolution, on the other hand, is measured from the distribution of residuals of muon hits with respect to the extrapolation of muon segments built using all available layers but the one under study.

\section{Muon detectors efficiency and resolution}

An unbiased hit efficiency can be measured only in DT and RPC chambers, as CSC cannot provide a readout without being triggered. The DT and RPC hit efficiencies are shown in Fig. 2: both are stable over time and in agreement with the expectations for the whole Run-2 [7, 8].

Fig. 3 shows the segment efficiency for the DT and CSC subsystems. The DT segment efficiency is stable against fluctuations in the single hit efficiency. Previous inefficiencies present during 2017 were due to readout failures of groups of channels, and were recovered in 2018 thanks to an improved front-end [7]. The CSC segment efficiency is high in both endcaps, typically larger than $90 \%$, as shown in 3, with few exceptions due to chambers with known localised hardware problems [9].

The resolution of DT chambers is of the order of $250 \mu \mathrm{m}$, and depends on the increasing crossing angle in the $r z$ projection, as shown in Fig. 4. The behaviour of $r \phi$ - and $r z$-layers is different because of the different orientation of wires. The resolution in the most external station of each sector, MB4, 

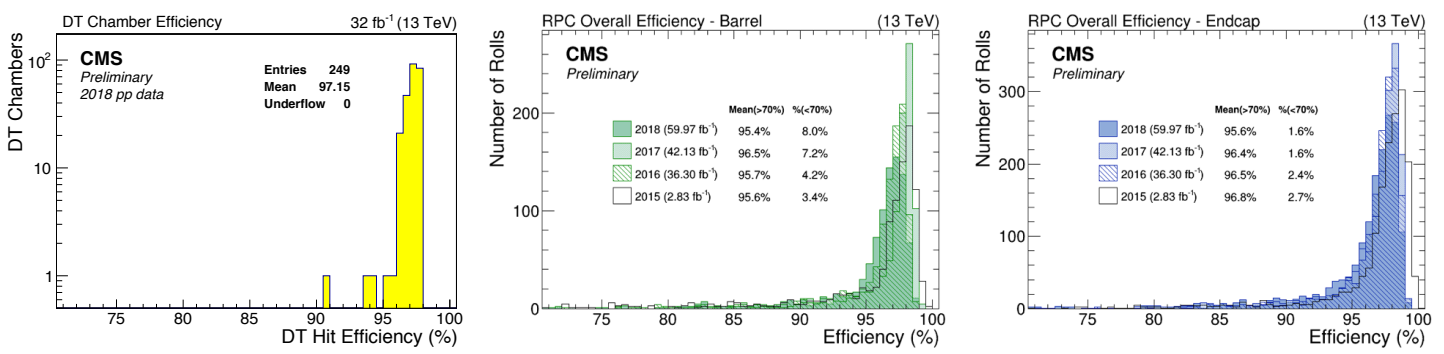

Figure 2. Single hit efficiency measured in the DT chambers (left, [7]) and in RPC chambers (barrel and endcap, respectively centre and right, [8]) during Run-2. The DT hit efficiency is shown for data collected in 2018, while the RPC hit efficiency is shown for the whole Run-2.
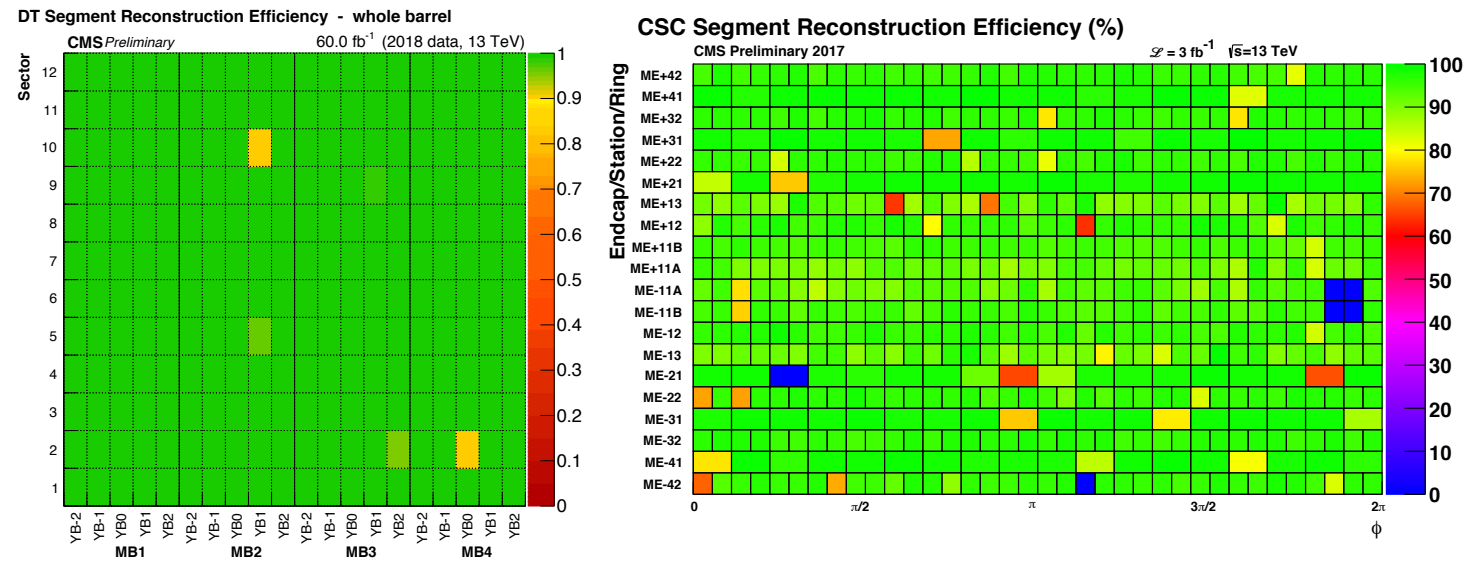

Figure 3. Local muon track segment efficiency measured in the DT chambers (left, [7]) and in CSC chambers (right, [9]) during Run-2. The DT segment efficiency is shown for data collected in 2018, while the CSC segment efficiency is shown for data collected in 2017.

is different: in fact, MB4 chambers do not have $r z$-layers, therefore it is not possible to correct the hit position in $r \phi$-layers for the propagation time of signals along wires. During 2018, the high voltage at the anode wire collecting the primary ionisation charge was lowered in order to mitigate the chamber aging and keep a high hit efficiency: chambers with a larger relative change in high voltage showed a larger change in resolution, which is indeed acceptable as it does not affect muon reconstruction and transverse momentum $p_{\mathrm{T}}$ assignment [7].

The CSC resolution, also shown in Fig. 4, is comprised between 40 and $140 \mu \mathrm{m}$, with variations dominated by non-uniformities of the magnetic field. The RPC resolution, shown in Fig. 5, is of the order of $1 \mathrm{~cm}$, dominated by the coarse segmentation of the cathodes, and computed from the extrapolation of the closest DT or CSC segment [10].

A crucial parameter for muon reconstruction is the time resolution: in the DTs it is obtained from a three-parameter fit to the segment, given that the hit position is measured from drift time conversion. The combination of up to 4 segments allows to reconstruct a standalone muon track, featuring a time resolution of approximately $1.5 \mathrm{~ns}$, as shown in Fig. 6. The distribution is slightly asymmetric because of the front-end dead time induced by delta rays, i.e. secondary electrons, or muon electromagnetic showers resulting in anticipated hits. Time resolution in the DT system 

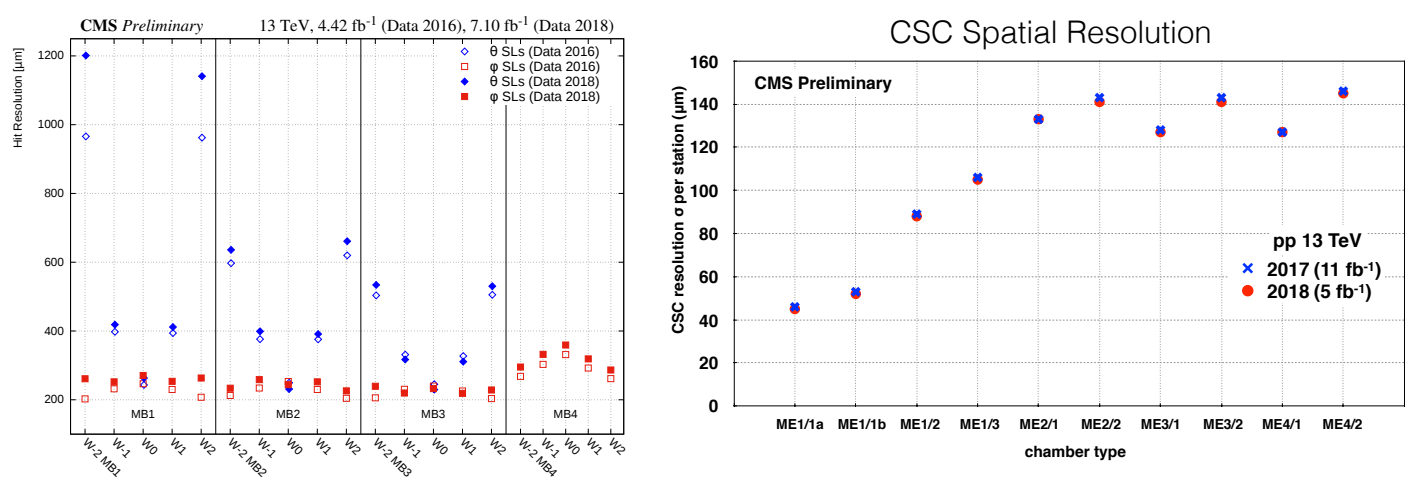

Figure 4. Muon hit resolution measured in the DT chambers (left, [7]) and in the CSC chambers (right, [7]) during Run-2. The DT hit resolution is shown for data collected in 2018 and 2016, while the CSC hit resolution is shown for data collected in 2018 and 2017.
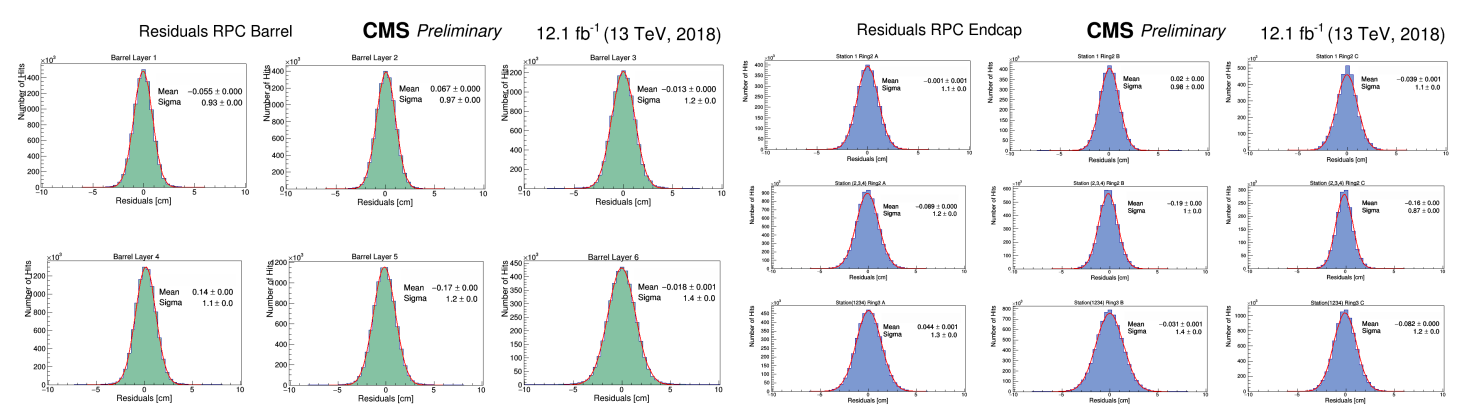

Figure 5. Muon hit resolution measured in the RPC chambers (barrel and endcap, respectively left and right, [10]) during Run-2, shown for data collected in 2018.

is unaffected by the high-voltage change [7]. The RPC front-end currently allows for a coarse granularity time measurement, therefore the time resolution is expressed in terms of differences in the bunch crossing assigned by RPC hits with respect to the bunch crossing assigned to the event [10]. Misassignment is below 1\%, as shown in Fig. 6.

The CSC cathode time is obtained from a template fit to the cathode pulse, while the CSC anode time is obtained from the match to the closest-in-time cathode time, in a window built around the trigger time, resulting in a discretized distribution. The CSC segment time is found by averaging over 6 hits after proper calibration, and the overall figure of merit is referred to standalone endcap muon tracks, obtained combining up to 4 CSC stations, featuring a time resolution of approximately $2.1 \mathrm{~ns}$, as shown in Fig. 7 [10].

\section{Performance of muon triggers during Run-2}

Muon triggers have been crucial in the CMS physics program. The Level-1 (L1) muon trigger was upgraded after Run-1, from a sub-detector-oriented approach to a geographical approach [11]. Its current version features an early exploitation of the redundancy of all three muon detection systems in the trigger process, in order to cope with increased rates at $13 \mathrm{TeV}$ proton-proton collision energy and instantaneous luminosities 50 to $100 \%$ larger than the LHC design luminosity 

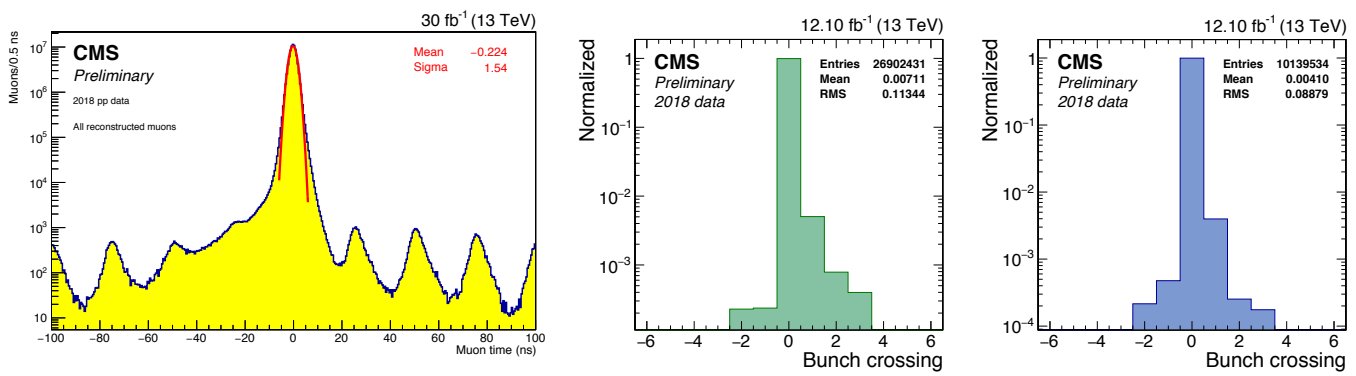

Figure 6. Muon time resolution measured in the DT chambers (left, [7]) and in RPC chambers (barrel and endcap, respectively middle and right, [10]) during Run-2, shown for data collected in 2018.
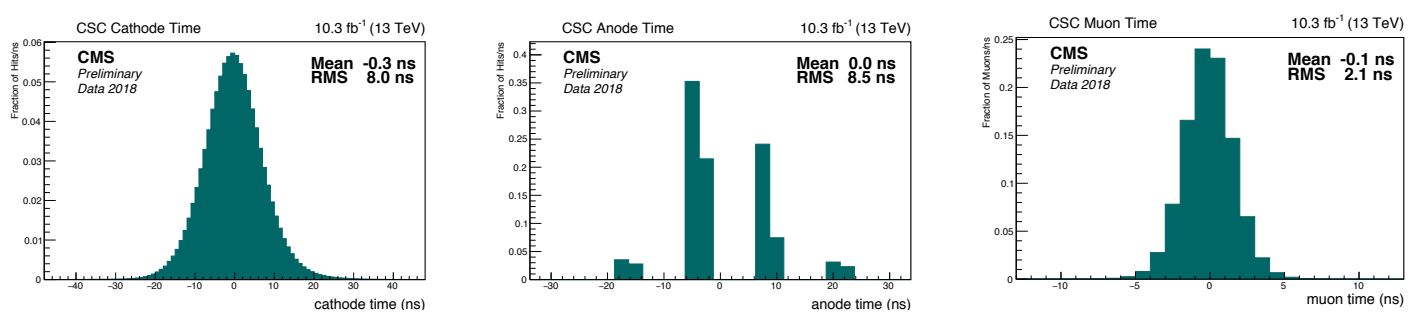

Figure 7. Muon time resolution measured in the CSC chambers during Run-2, shown for data collected in 2018: cathode time (left), anode time (middle) and CSC standalone time (right) [10].

$\mathcal{L}=10^{34} \mathrm{~cm}^{-2} \mathrm{~s}^{-1}[11,12]$. Muon trigger primitives, i.e. online-built track segments, and clusters of RPC hits, are combined before entering the muon track finder, which currently comes in three flavours dedicated to each barrel/endcap/overlap region. Trigger primitives from the DT and RPC systems are combined into a single trigger primitive by the TwinMux concentrator [13]. Cleaning and sorting are performed by the global muon trigger, which keeps up to 8 candidate muons per event. Fig. 8 shows the most important effect of this approach: while keeping a high L1 muon tracking efficiency, about $95 \%$ on average above threshold, the rate has been reduced w.r.t. Run-1 trigger by $10 \%$ to $80 \%$ depending on track rapidity $[14,15]$.

Fig. 9 shows the effects of combining DT and RPC trigger primitives. The fraction of out-oftime DT trigger segments is reduced using the better bunch crossing identification of RPC clusters, an average increase of the trigger primitive generation efficiency of about $1.4 \%$ is measured. Also, RPC-only segments have been included in the TwinMux since 2017: the barrel muon track finder shows up to $2 \%$ higher efficiency in the crack regions between wheels, and a trigger rate reduction of $3 \%$ at $p_{\mathrm{T}}>25 \mathrm{GeV}$, due to the improved momentum assignment $[16,17]$.

\section{GEM slice test during Run-2}

The GE1/1 system is the first of the CMS Phase-II Upgrade projects. The muon system will be complemented by triple GEM detectors in the very forward region, $|\eta|>1.6$, where the occupancy is high, in order to enhance both trigger and reconstruction, because of their high spatial resolution, rate capability and radiation-tolerant technology [4]. These detectors will be arranged in 36 superchambers per side. Five such super-chambers were installed in front of the endcap return yoke, on the side of CMS corresponding to exit point for protons coming from the Jura mountains to LHC 

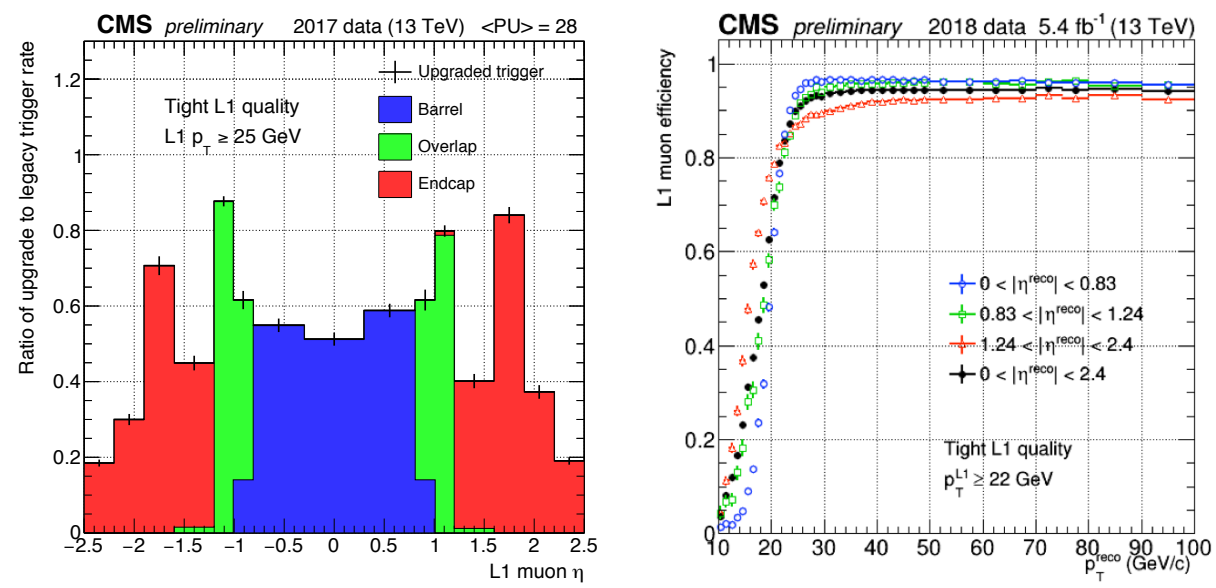

Figure 8. Effect of the upgraded L1 muon trigger on trigger rate measured in Run-2, in comparison to Run-1, shown for data collected in 2017 (left, [15]). Muon trigger efficiency for a $p_{\mathrm{T}}=22 \mathrm{GeV}$ threshold for the different regional track finders measured in Run-2, shown for data collected in 2018 (right, [14]).
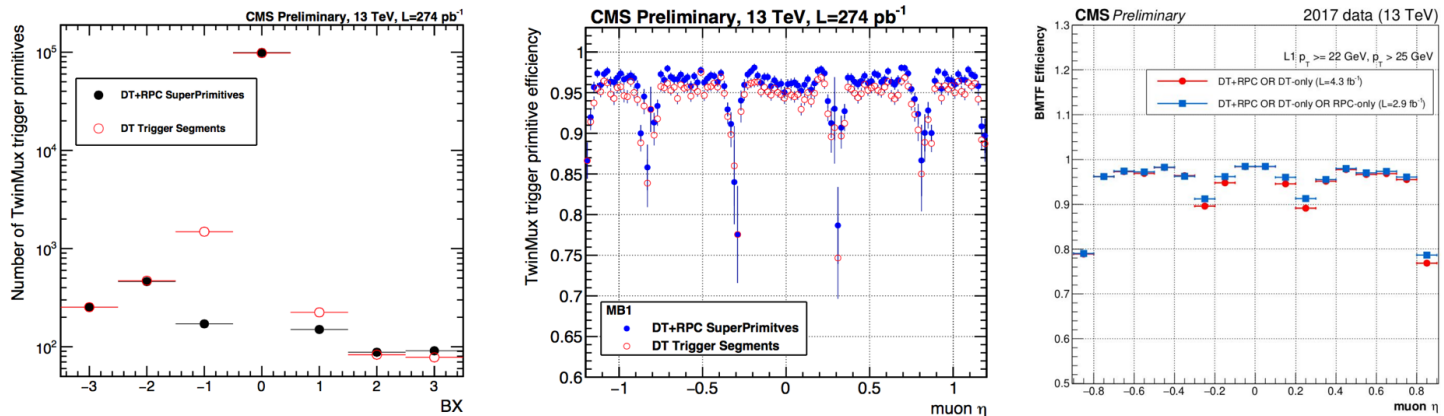

Figure 9. Time distribution of standalone DT trigger primitives and combined DT+RPC trigger primitives (left, [17]). Efficiency of standalone DT trigger primitives and combined DT+RPC trigger primitives in the innermost muon station MB1 (centre, [17]). Barrel muon track finder efficiency before and after the introduction of standalone RPC trigger primitives in the TwinMux concentrator (right, [16]).

Point 5, to collect data in 2017 and 2018: four were used to test the GEM system rate capabilities, and one to evaluate alternative electronics for the readout and high-voltage supply. The completion of the GE1/1 project is expected by Winter/Spring 2020.

This slice test confirmed that GEM efficiency is higher than $90 \%$, and comparable with other muon detectors, with exceptions due to front-end thresholds tuning. GEM clusters group a few strips, typically 1 to 3, consistent with test beam measurements, and with an angular resolution of the order of $10^{-4}$ radians, as shown in Fig. 10. It was observed that the front-end ASIC suffered irreversible channel losses, attributed to propagating discharges from the third GEM foil to the anode plane. Such effect is expected to be mitigated with the new front-end ASIC design, featuring a discharge damage probability reduction from $\approx 93 \%$ to $\approx 3 \%[18]$. 

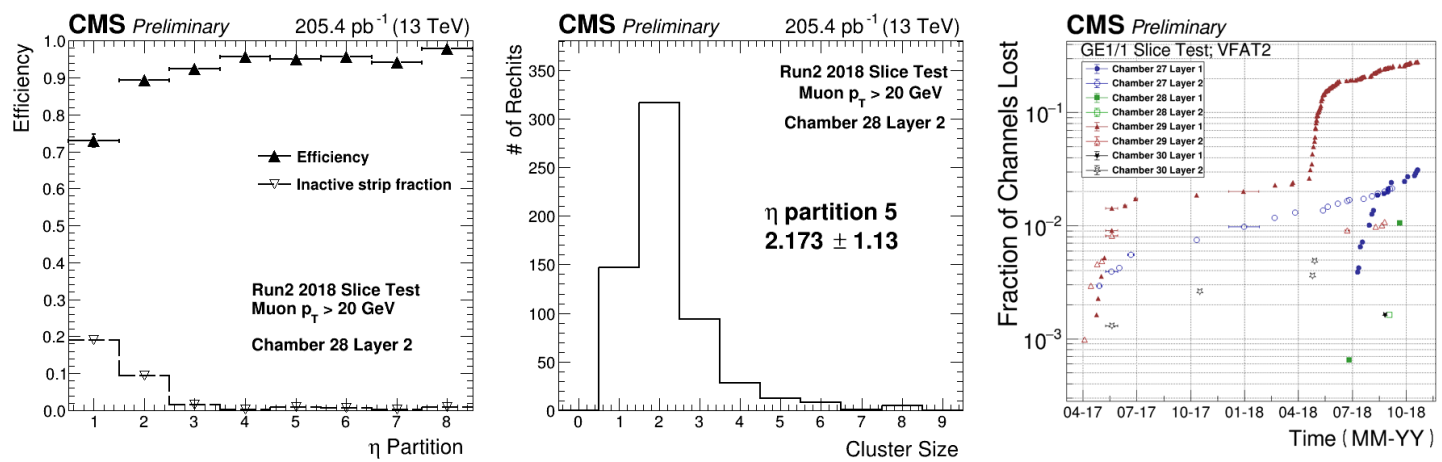

Figure 10. Example of the measured muon hit efficiency with GEM detectors (left) and example of GEM cluster size (centre) during Run-2. Channel losses in GEM detectors during Run-2 as a function of time (right).

\section{Conclusions}

The CMS muon system has been thoroughly monitored throughout the whole Run-2, and all subdetectors are found to cope with the design requirements after long operations. The trigger and reconstruction efficiency and resolution are stable and comparable, if not better, to the Run-1 legacy figures, allowing to collect valuable data for physics searches, precision measurements, and detector health monitoring.

Future challenges have already been tackled: the first tests of muon system upgrade with new GEM detectors in the forward region are promising, while other activities, such as longevity studies and development of novel triggers, are progressing fast.

\section{References}

[1] CMS Collaboration, The CMS experiment at the CERN LHC, JINST 3 (2008) S08004.

[2] CMS Collaboration, Observation of a new boson at a mass of $125 \mathrm{GeV}$ with the CMS experiment at the LHC, Phys. Lett. B 716 (2012) 30-61.

[3] CMS Collaboration, Measurement of the $B_{s} \rightarrow \mu^{+} \mu^{-}$branching fraction and search for $B^{0} \rightarrow \mu^{+} \mu^{-}$ with the CMS Experiment, Phys. Rev. Lett. 111 (2013) 101804.

[4] CMS Collaboration, CMS Technical Design Report for the Muon Endcap GEM Upgrade, CERN-LHCC-2015-012, CMS-TDR-013.

[5] CMS Collaboration, Performance of the CMS muon detector and muon reconstruction with proton-proton collisions at $\sqrt{s}=13 \mathrm{TeV}$, JINST 13 (2018) P06015.

[6] CMS Collaboration, The Phase-2 Upgrade of the CMS Muon Detectors, CERN-LHCC-2017-012, CMS-TDR-016.

[7] CMS Collaboration, Performance of the CMS Drift Tubes at the end of LHC Run 2 CMS Detector Performance Note CMS-DP-2019-008, https://cds.cern.ch/record/2673608.

[8] CMS Collaboration, RPC DPG Approved Results for Conferences, (2019) https://twiki.cern.ch/twiki/bin/view/CMSPublic/RPCPlots.

[9] CMS Collaboration, CSC Performance in 2017 CMS Detector Performance Note CMS-DP-2017-038. 
[10] CMS Collaboration, Performance of CMS muon detectors in 2018 Collision Runs CMS Detector Performance Note CMS-DP-2018-047, https://cds.cern.ch/record/2630439.

[11] CMS Collaboration, CMS Technical Design Report for the Level-1 Trigger Upgrade, CERN-LHCC-2013-011, CMS-TDR-12.

[12] A. Triossi et al., The CMS Barrel Muon trigger upgrade, JINST 12 (2017) C01095.

[13] A. Triossi et al., A New Data Concentrator for the CMS Muon Barrel Track Finder - Phase I Upgrade, PoS(TIPP2014)412.

[14] CMS Collaboration, Level-1 Muon Trigger Performance CMS Detector Performance Note CMS-DP-2018-044, https://cds.cern.ch/record/2629852.

[15] CMS Collaboration, Level-1 muon trigger performance in 2017 data and comparison with the legacy muon trigger system CMS Detector Performance Note CMS-DP-2017-041, https://cds.cern.ch/record/2286327.

[16] CMS Collaboration, The inclusion of RPC only segments in the Barrel Muon Track Finder CMS Detector Performance Note CMS-DP-2018-007, https://cds.cern.ch/record/2305548.

[17] CMS Collaboration, Performance of the CMS TwinMux Algorithm in late 2016 pp collision runs CMS Detector Performance Note CMS-DP-2016-074, https://cds.cern.ch/record/2239285.

[18] CMS Collaboration, GE1/1 Slice Test Performance CMS Detector Performance Note CMS-DP-2019-010, https://twiki.cern.ch/twiki/bin/view/CMSPublic/GEMDPGPublic. 